\title{
Comportamiento de precios de mercados y análisis de volatilidad, para durazno (Prunus persica [L.] Batsch.) producido en Boyacá, Colombia
}

\section{Behavior of market prices and analysis of volatility for peach (Prunus persica [L.] Batsch.) produced in Boyaca, Colombia}

ANDRÉS GONZÁLEZ G. ${ }^{1,4}$

GLORIA A. PUENTES M. ${ }^{2}$

EDGAR F. RUIZ R. ${ }^{3}$

Duraznos en el Central Comercial de Mercado Minorista de Duitama, Boyacá.

Foto: A. González G.

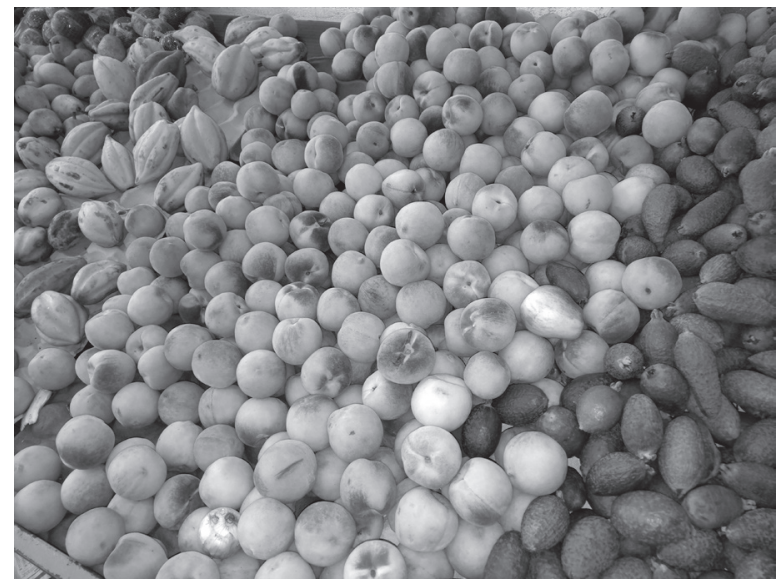

\section{RESUMEN}

En la actualidad, se encuentran diferentes estudios de caducifolios, y en este caso de durazno en temas como producción, cosecha, poscosecha, mercados. Sin embargo al desarrollar la formulación de los proyectos de inversión, no se conoce el comportamiento del precio de la fruta en los últimos años, en los mercados más representativos del país. Es así como se desarrolló esta investigación centrada en el análisis de los precios de durazno en tres mercados de Colombia: (a) Central de Abastos de Bogotá, (b) Mayorista de Antioquia, Medellín y (c) Reten sur de Tunja, con el objetivo de comprobar si durante el periodo 2006 al 2011, se ha presentado estacionalidad y volatilidad en los precios de mercados, más aun cuando los pequeños productores manifiestan que cada vez este agronegocio es menos rentable y al elaborar nuevos proyectos para invertir en esta especie, se presenta la dificultad en la asignación de los precios de venta. Este aspecto incrementa el riesgo en la toma de decisiones para los productores. El estudio inició desde el análisis de las generalidades de la producción de caducifolios y del durazno, producto que se concentra principalmente en el departamento de Boyacá, Colombia, en agroempresas de tipo minifundistas, de economía campesina. Continuó con la relación de datos de precios por kg/mes, entre el año 2006 y 2011. En los tres mercados, la metodología utilizada fue un análisis exploratorio de los precios por medio del Análisis Espectral Singular, utilizando el Método de Loess. Además, se empleó la metodología de Box y Jenkins. Se logró determinar el comportamiento de tendencia y estacionalidad en los precios del durazno. De otra parte, se identificó que el comportamiento estacional y de tendencia no está presente en los mercados Corabastos de Bogotá y Central mayorista de Antioquia, si se encontró estacionalidad en el mercado de Tunja Cabe resaltar que la volatilidad de los precios no se observó en

\footnotetext{
Facultad de Ciencias Económicas, Universidad Nacional de Colombia, Bogotá (Colombia).

Facultad Seccional Duitama, Programa Administración de Empresas Agropecuarias, Universidad Pedagógica y Tecnológica de Colombia, Duitama (Colombia).

3 Departamento de Ciencias Básicas, Universidad Santo Tomás, Seccional Tunja, Tunja (Colombia).

4 Autor para correspondencia.ndgonzalezgon@unal.edu.co
} 
los tres mercados estudiados, se determinó que el precio promedio actual de duraznos per kg en el mercado de reten sur Tunja es de $\$ 1.800$ (USD 0,60) y de $\$ 3.500$ (USD 1,17) en los mercados de Bogotá y Medellín.

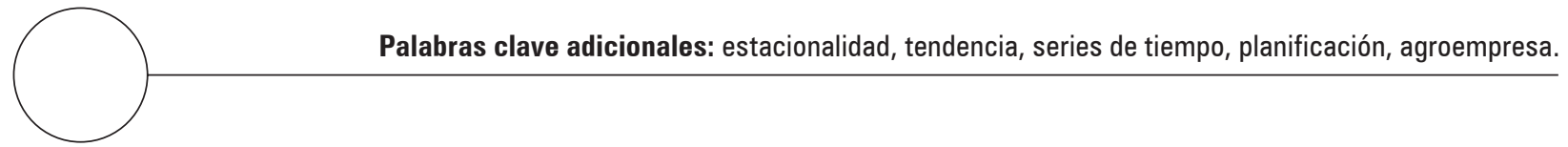

\section{ABSTRACT}

At present, there are different studies on deciduous fruits, including the peach, for subjects such as production, harvest, postharvest, and markets. However, for developing the formulation of investment projects, the behavior of fruit prices in the most representative markets of the country are unknown for recent years. This research focused on the analysis of peach prices in three markets in Colombia: (a) Central de Abastos of Bogota, (b) Wholesaler of Antioquia, Medellín and (c) Reten Sur de Tunja, with the objective of verifying whether seasonality and volatility in market prices have occurred during the period 2006 to 2011, even more so when small producers report that agribusiness is becoming less profitable. When new projects are developed to invest in these species, difficulty in the allocation of sales prices is seen. This increases risk in decision making for producers. The study began with the analysis of the generalities of production of deciduous fruits and peaches, a product that is concentrated mainly in the department of Boyacá, Colombia, in agribusinesses of smallholders with a rural economy. It continued with the relation of data of prices per $\mathrm{kg} / \mathrm{month}$ between the years 2006 and 2011. In the three markets, the methodology used an exploratory analysis of the prices by means of the Singular Spectral Analysis, along with the Loess Method and the Box and Jenkins methodology. It was possible to determine the trend behavior and seasonality in the peach prices. On the other hand, it was observed that a seasonal and trend behavior was not present in the markets of Corabastos of Bogota or Central Wholesale of Antioquia, but seasonality was seen in the market of Tunja. It should be emphasized that the price volatility was not observed in the three markets; it was determined that the current average price per $\mathrm{kg}$ of peach fruits in the Reten Sur de Tunja market is $\$ 1,800$ Colombian Pesos (USD 0.60) and $\$ 3,500$ Colombian Pesos (USD 1.17) in the Bogotá and Medellin markets, respectively.

Additional key words: seasonality, trend, time series, planning, agribusiness.

Fecha de recepción: 10-02-2017 Aprobado para publicación: 15-04-2017

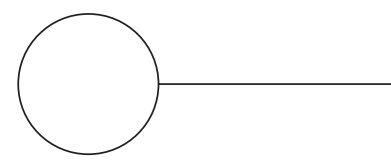

El término "caducifolios" hace referencia a plantas que pierden sus hojas en ciertas épocas del año, dado su origen subtropical (Cárdenas y Fischer, 2013) y los mismos autores anotan que entre los frutales caducifolios se distinguen las pomáceas (frutales de pepita) y las drupáceas (frutales de hueso); las drupáceas más importantes son los durazneros (Prunus persica L. Batsch.) y los ciruelos (japonés: Prunus salicina Lindl. y el europeo: Prunus domestica L.). Los durazneros, originarios de China (Westwood, 1993), producen frutos de tipo drupa con una forma globosa u ovoide, y diámetros entre 4 y $10 \mathrm{~cm}$ (Baugher, 2003).

El área cosechada de frutales caducifolios en Colombia para el año 2010 fue de 52.984 ha (FAOSTAT, 2012). La producción porcentual mundial de duraznos-melocotón, ciruelas, peras y manzanas, para el año 2010 fue 38,$7 ; 26,9 ; 31,1$ y $3,2 \%$, respectivamente; donde, los principales aumentos porcentuales en volumen de producción en el año 2010 fueron de melocotón y durazno $(77 \%)$, pera $(75 \%)$ y manzana (64\%), en comparación al volumen de 2009 (FAOSTAT, 2012).

Miranda et al. (2013) anotan que, en la actualidad, las zonas de producción están dispersas por diferentes ecorregiones del país, con diversidad de cultivares en su mayoría de procedencia desconocida, con niveles de uso de tecnología variables entre las regiones y entre las mismas fincas. Los sistemas de producción, de igual manera, presentan variaciones y niveles contrastantes de desarrollo. 
Los departamentos representativos en el cultivo de durazno son Boyacá $60,5 \%$ del volumen de producción, Cundinamarca 10,2\%, Santanderes 29,1\% y Meta $0,2 \%$, con un área aproximada de 1.490 ha. y una producción promedio de $19.849 \mathrm{t}_{\text {año }}{ }^{-1}$ para el 2011 (Minagricultura, 2012), Actualmente, los retos para los cultivos de frutales caducifolios están relacionados con su capacidad competitiva, frente a la fruta importada, teniendo en cuenta el control de plagas y enfermedades del cambio climático, la necesidad de reducción el uso de plaguicidas y suministro, manejo de la fruta en precosecha y poscosecha con la necesidad para la logística de adaptarse a los diferentes mercados (Fachinello et al., 2011).

La importancia del departamento de Boyacá en la producción de caducifolios se fundamenta en avances importantes en el modelo de manejo empresarial, introducción de nuevas variedades, innovaciones tecnológicas, implementación de infraestructura de riego y drenaje, trabajo asociativo, incremento en el área cosechada, planeación de la producción según al mercado, y consolidación de la cadena productiva.

Se destaca como práctica fundamental la programación de las cosechas, que permite oferta de producto en periodos de escasez con mejores precios de mercado (Puentes, 2006).

Las Unidades productivas de caducifolios en el Departamento de Boyacá, reúnen las siguientes características: área en promedio de 3,72 ha, altas pendientes $18,29 \%$ en el $62 \%$ de las fincas, con mínimo 0,48 ha de la finca dedicada a este cultivo, 133 árboles/finca, edad promedio de 7,79 años, rendimiento promedio de 27,47 tha-1 (Puentes et al., 2008),

De los sistemas analizados, se destaca la ausencia de modelos de gestión empresarial, en los que se realizan altas inversiones en la implementación de los cultivos y en asistencia técnica (Puentes et al., 2008), asumiendo altos riesgo sin la suficiente información para la toma de decisiones, por lo tanto se desarrollan en entornos de alta incertidumbre (Zuluaga y Madrid, 2010), asociados factores intrínsecos de la producción, condiciones climáticas, costo de los insumos y en especial a la volatilidad de los precios en los mercados.

Según la Organización Mundial para la Alimentación y la Agricultura (FAO, 2012) se considera que los precios mundiales de alimentos de origen agropecuario presentan una tendencia al alza, siendo un factor de preocupación para la comunidad internacional, poniendo en riesgo la seguridad alimentaria.

En investigaciones hechas sobre inflación y volatilidad en mercados agropecuarios, el Comité de Seguridad Alimentaria Mundial (CFS, 2011) resalta principalmente tres puntos básicos: la excesiva volatilidad de los precios, crisis alimentaria cíclica y estacional y situaciones emergentes de escases, de ahí la urgencia de implementar políticas que garanticen el acceso a alimentos a la población más vulnerable.

Según Aimin (2010), los productores acuden a estrategias de diversificación de su oferta para minimizar los riesgos, en busca de asegurar un nivel medio de ingresos; pero existen serias deficiencias en la información disponible, así como en la calidad de la misma; esto limita la capacidad de identificar las mejores opciones de inversión por parte de los cultivadores, llevándolos a tomar decisiones en entornos de alta incertidumbre. La ausencia de análisis estadístico de producción y comportamiento de los precios de los productos en los mercados incrementan el riesgo (Taušer y Čajka 2014).

El aporte del presente estudio es la generación de modelos econométricos que permitan elaborar pronósticos del comportamiento de los precios de durazno en distintos escenarios, con simulación estadística para suministrar información confiable que facilite la predicción de comportamientos de precio, aplicable a la gestión de los agroempresarios y así tomar las mejores decisiones, dado que se minimiza la incertidumbre sobre el retorno de las inversiones en cultivos de caducifolios en el departamento de Boyacá, siendo un modelo que puede ser aplicable a otros productos agropecuarios.

De ahí la importancia del estudio sobre los efectos de la volatilidad y su relación con la inflación en los mercados de alimentos de origen agrícola, a fin de mejorar la capacidad de acceso a la información de comportamiento de precios por parte pequeños productores y lograr que se convierta en insumo indispensable en el proceso de toma de decisiones de inversión los agronegocios.

Esta información es valiosa para los agroempresarios de caducifolios, para llevar a cabo la gestión en cada una de las áreas del negocio y de acuerdo con las 
etapas y actividades productivas de durazno (Puentes, 2006).

Los caducifolios, por ser cultivos permanentes, poseen características muy definidas en su modelo de flujo de ingresos y egresos, con inversiones a largo plazo, generando ingresos a partir del tercer año, que van aumentando hasta el décimo, luego se estabilizan y vuelven a decrecer en los 25 o 30 años (Castro y Puentes, 2012), el precio de venta por kg producido y su comportamiento en el tiempo son factores determinantes en la viabilidad financiera.

El reducir los costos de transacción y los riesgos en los mercados de alimentos básicos puede acelerar el crecimiento y beneficiar a los pobres. La volatilidad de los precios de alimentos básicos incide en el gasto de los consumidores de estratos bajos (FAO, 2006).

Los altos riesgos de volatilidad de los precios son persistentes tanto para los agricultores como para los consumidores $\mathrm{y}$, por ende, es importante contar con redes de protección (CEPAL et al., 2011).

La volatilidad es definida como los cambios abruptos de sentido y magnitud de los precios, en el tiempo, situación que resulta perjudicial dado que puede generar inflación por el incremento del riesgo del qué y cómo producir alimentos (FAO, 2010), como respuesta los diferentes agentes del mercado que asumen un mayor riesgo elevan los precio, generando incertidumbre entre los productores y consumidores, lo cual reduce la confianza y afecta de manera negativa los niveles de comercio e inversión (Andreyeva et al, 2010).

Los estudios de comportamientos de precios en alimentos de origen agrícola, han puesto en evidencia la presencia de fenómenos de volatilidad en el periodo comprendido entre los años 2008 a 2009, en especial de commodities (Gilbert y Morgan, 2010), La volatilidad afecta de manera significativa a los más pobres, generando la inconformidad social al limitarse el acceso de alimentos de la canasta básica (Bellemare, 2015). Es importante aclarar que la volatilidad de precios de los alimentos no es una problemática de productores y consumidores, concierne también a los gobiernos y su política económica.

El conocimiento de la volatilidad se ha concentrado en los mercados financieros y cambiarios, tomando una gran fuerza como área de estudio en las últimas décadas, por ser un insumo para la evaluación de riesgos, la mayor parte de las investigaciones se concentran en su evolución y dinámica; el tema desde la econometría aborda dos tipos de modelos: los modelos de heterocedasticidad condicional autorregresiva (ARCH), propuestos por Engle (1982) y los modelos (GARCH), planteados por Bollerslev (1986) con todas las variantes que a partir de ellos han ido surgiendo los modelos de volatilidad estocástica [modelos (SV), propuestos por Taylor (1986)

De estos modelos, los más utilizados han sido los modelos ARCH, ya que son los más sencillos de estimar, dado que están implementados en la mayoría del software econométrico. En los últimos años se ha incrementado el número de investigaciones acerca de la incidencia de la volatilidad en los precios de los commodities, por ser considerados como alternativas de inversiones diferentes a los activos financieros. Esta situación, según Doporto y Michelena (2011), está asociada a la política monetaria de los Estados Unidos, que propone tasas de interés cercanas a cero; la posibilidad de los commodities de diversificar los riesgos de los portafolios de inversión al poseer correlación con bonos y las acciones; la depreciación del dólar respecto de otras monedas y capitales que buscan mayores rentabilidades, entre otras.

Cooper et al. (1975) aseguran que la volatilidad surge de los impactos (shocks) que se originan en el mercado, los cuales pueden estar o no correlacionados (Gilbert y Morgan, 2010), afirman que este parece haber sido el caso en 2007-2008, cuando la mayoría de los precios agrícolas y precios de productos no agrícolas (energía, metales y tarifas de carga) se elevaron simultáneamente en este periodo.

En el estudio de Balcombe (2009) aplicando dos métodos econométricos para explorar la naturaleza y causas de la volatilidad de los precios de los productos básicos agrícolas en el tiempo, concluyó que la volatilidad de un determinado precio es dependiente de las reservas, los rendimientos, la concentración de exportación y su relación con la volatilidad de otros precios, incluyendo los precios del petróleo, las tasas de cambio y las tasas de interés.

En el ámbito latinoamericano, entidades como la CEPAL et al. (2011 y los autores citados en este), informan que estudios realizados en los últimos años identifican la crisis con un carácter estructural, siendo más que aspecto coyuntural el alza de precios observados en el periodo 2007-2008 lo que amplió la visión sobre el comportamiento de los mercados de 
productos agrícolas y su volatilidad a largo plazo, en donde también se analizaron un conjunto de factores homogéneos identificados como causas potenciales de la crisis.

En el presente estudio se planteó como objetivo evaluar el comportamiento de precios de mercados mayoristas de durazno en el periodo 2006 a 2011, para identificar la tendencia, de estacionalidad y volatilidad de los mismos.

\section{METODOLOGÍA}

El estudio se basó en una investigación documental y en un análisis econométrico, aplicando una metodología de tipo correlacional, entendiendo las posibles interacciones de sentido entre la variable independiente precio y las dependientes tendencia, estacionalidad.

Lo anterior con el objetivo de determinar la tendencia, estacionalidad en los precios y su volatilidad dentro de las series históricas; en primer lugar, se identificaron las principales centrales mayoristas del país, que comercializan durazno cultivado en Boyacá; se recurrió a la información estadística del Sistema de Información de Precios y Abastecimiento del Sector Agropecuario (SIPSA), en el componente de precios mayoristas, el cual captura información de productos frescos en 57 mercados de 20 departamentos.

Para el análisis de precio, se identificaron los mercados más representativos para el durazno, teniendo en cuenta la continuidad en la oferta, es así como se seleccionaron tres centrales mayoristas que comercializan durazno, Bogotá (Corabastos), Medellín (Central Mayorista de Antioquia) y Tunja (Reten Sur). Siendo estos los mercados que presentaron el mayor número de datos de precio por $\mathrm{kg} / \mathrm{mes}$, en el periodo analizado (Agronet y SIPSA, 2015).

Se estandarizo una base de datos con 72 valores de precios en $\mathrm{kg} / \mathrm{mes}$ en cada mercado, por cada uno de los tres mercados relacionados por producto, durante 6 años, para un total de 216 observaciones. Con esta información se construyó un modelo econométrico estimativo del precio del durazno. A continuación, las series de tiempo se normalizaron y analizaron de la siguiente manera. Se partió de la revisión bibliográfica para establecer la fundamentación teórica de técnicas exploratorias, tomando como base, el Análisis Espectral Singular (AES), según Ramírez et al. (2012), y complementándolo con el método de Loess de acuerdo con Giraldo (2006) para identificación de tendencias, se usó la función stl()y el AES con paquete Rssa del software libre R-project.

Por medio de exploración se pudo establecer el componente de tendencia, estacionalidad e irregularidad de cada una de las series de tiempo de los precios en cada uno de los mercados mayoristas. Luego se aplicó la metodología de Box-Jenkins (Fig. 1), para explicar el comportamiento de los precios del durazno en los mercados seleccionados; los pasos a desarrollar en cada uno de los modelos fueron:

Primero, se categorizaron los datos según su origen para cada una de las especies en cada mercado mayorista, luego se normalizaron los datos de precios de $\mathrm{kg} / \mathrm{mes}$, se estiman los datos faltantes en cada una de las series de tiempo con el promedio de los datos adyacentes en el periodo, se decidió no deflactar los precios ya que el Indice de Precios al Consumidor (IPC), para el renglón de alimentos no tuvo una variación significativa, y al ser aplicado suaviza en comportamiento de la serie de tiempo, lo cual afecta el comportamiento y sesga la información para su análisis.

Luego se realizó observación exploratoria (Fig. 2), por medio del Análisis Espectral Singular (AES) y método de Loess, de esta manera se identificó la existencia o ausencia de tendencia y estacionalidad en la serie de tiempo, de igual manera se verificó la validez del modelo elegido por la metodología de Box y Jenkins.

Se probaron modelaciones de tipo: AR, MA, ARMA, ARIMA Y SARIMA, seleccionando la más adecuada para la exploración de la serie, a continuación el modelo se ajustó para cada uno de los mercados a fin de observar gráficamente su comportamiento, aceptando el modelo para cada mercado que presenta el criterio de información de Akaike (AIC) con los valores más pequeños y que cumpliera con supuestos de varianza con errores no autocorrelacionados, normalidad y ruido blanco, requeridos para darle validez al modelo. 
MODELO DE ANÁLISIS DE SERIES TEMPORALES Y MODELOS AUTOPROYECTIVOS ESTOCÁSTICOS METODOLOGÍA DE BOX AND JENKINS

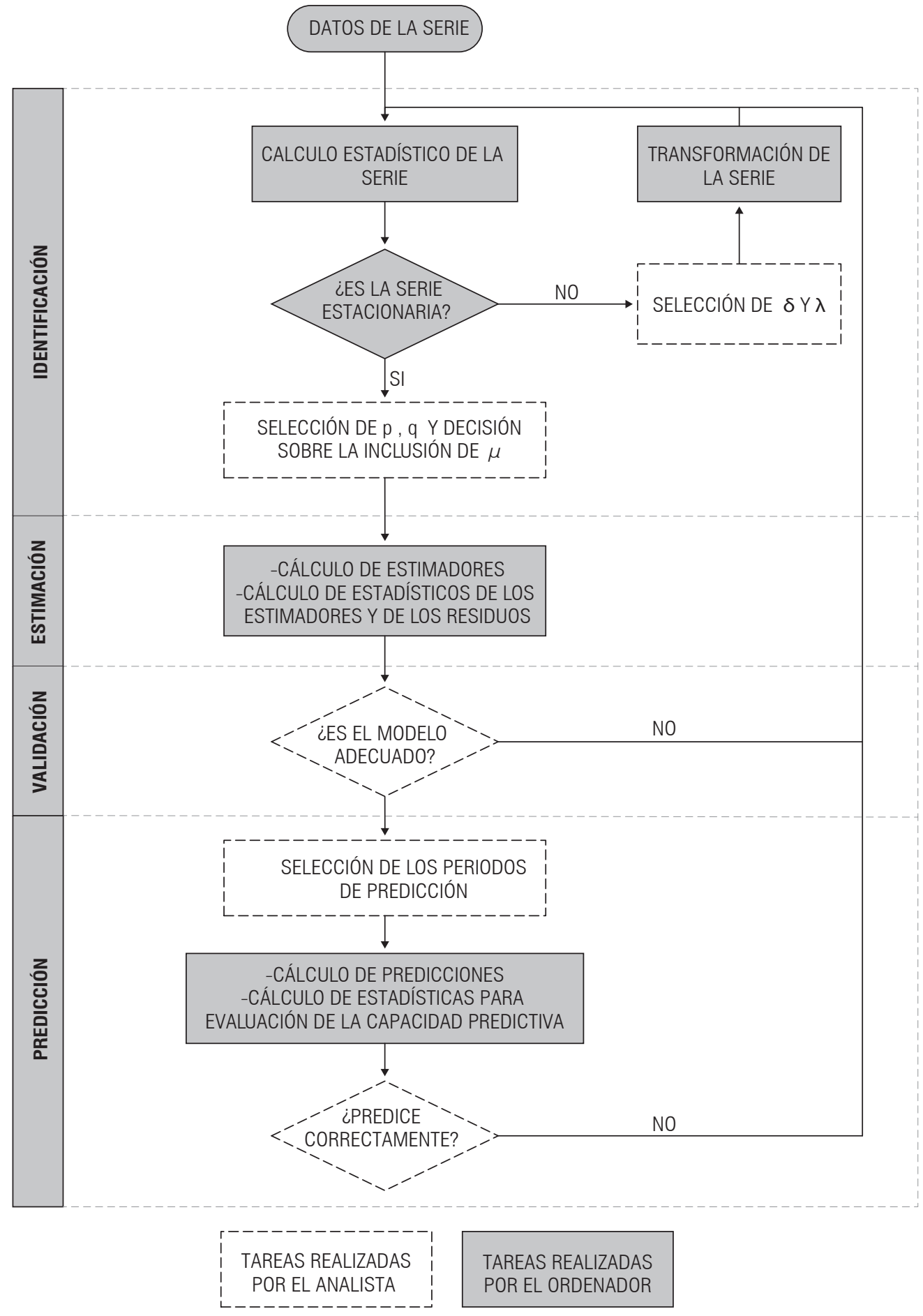

Figura 1. Flujograma aplicado para el procesamiento de las series de tiempo de precios de mercado mayorista, adaptado de Pérez (2006). 


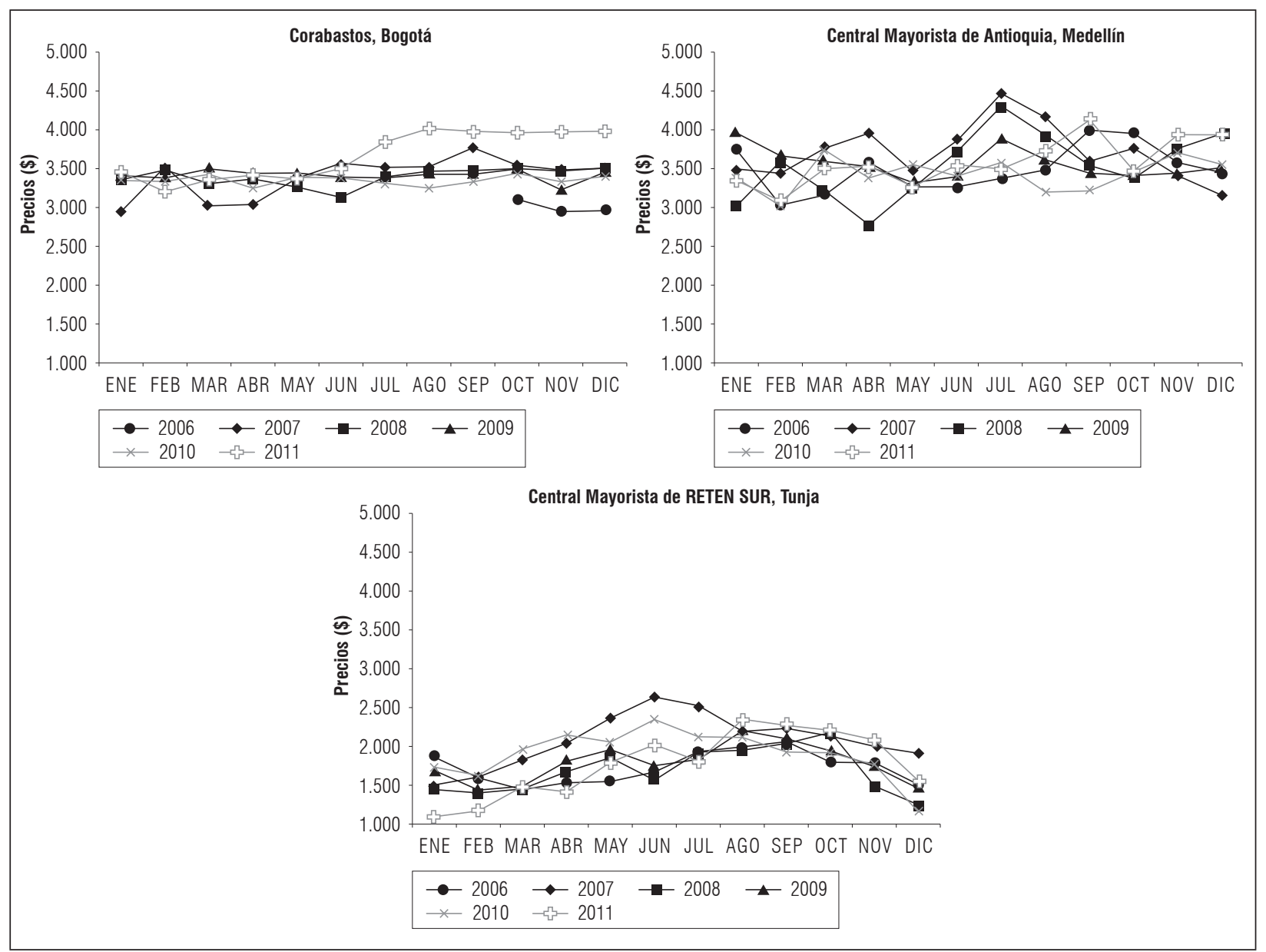

Figura 2. Comportamiento anual de precios de durazno, comparativo en los tres mercados analizados en el periodo 2006 a 2011.

\section{RESULTADOS Y DISCUSIÓN}

En términos generales la percepción de la estacionalidad y volatilidad en el precio de los alimentos de origen agrícola, no se cumple para el caso del durazno en los mercados objeto de estudio, el comportamiento histórico del precio del durazno para las tres centrales mayoristas identifico el comportamiento individual de cada central, Corabastos en Bogotá (Tab. 1), Central Mayorista de Antioquia en Medellín (Tab. 2). Así mismo se relacionan los precios del $\mathrm{kg}$ de durazno en el mercado más cercano a los centros de producción, el Reten Sur de Tunja, Boyacá.

En el análisis de las tablas anteriores se observa como en cada uno de los mercados, se mantienen precios con muy pocas diferencias y es importante resaltar la diferencia de precios de $\mathrm{kg}$ de durazno en los tres casos, notándose un precio promedio de $\$ 1.800$ (USD 0,62) en el mercado Reten Sur de Tunja, siendo el precio más bajo con relación a los otros dos; los mercados de Antioquia y Bogotá tienen comportamientos similares, obteniendo un precio promedio por $\mathrm{kg}$ de $\$ 3.584$ y $\$ 3,422$ ( USD 1,24 y 1,18 ), respectivamente (Tab. 4), la tasa de cambio empleada para la conversión a dólares es $\$ 2.900 \mathrm{COP} /$ \$ USD, resultado del promedio reportado por el Banco de la República en el periodo comprendido entre enero del 2015 a abril de 2017.

Se identificó que los precios del durazno son homogéneos en los tres mercados analizados, presentando mayor variabilidad en el mercado de Tunja. Es relevante poner en evidencia que los mercados grandes presentan una mayor homogeneidad en sus datos en relación a los mercados pequeños, esto es debido a que poseen una oferta de durazno más constante con relación a los mercados pequeños. Igualmente, el mercado Plaza Reten Sur de Tunja, el cual es el más cercano a las zonas de producción presenta el precio 


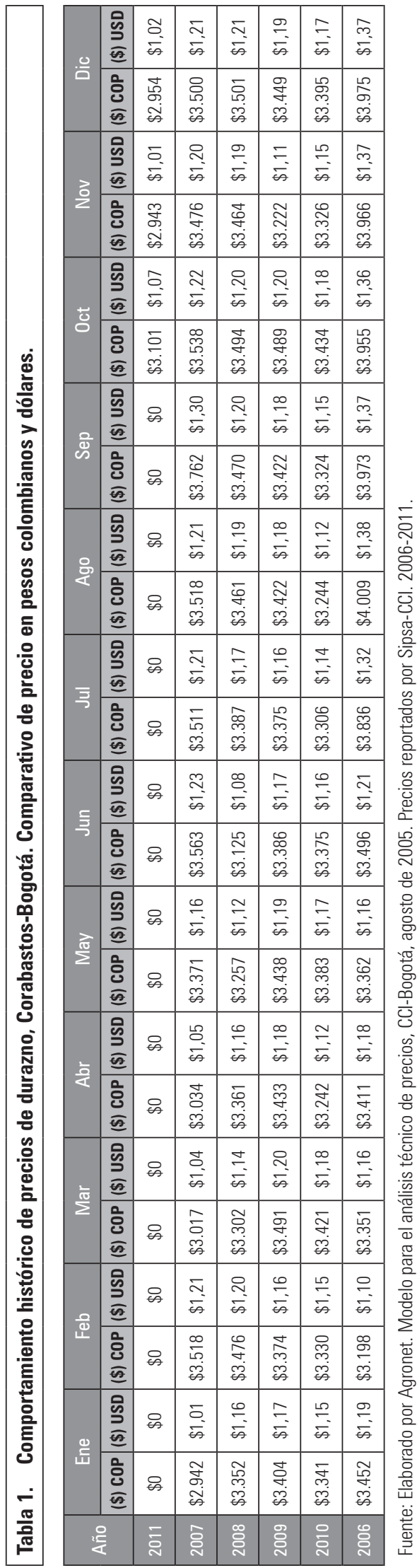

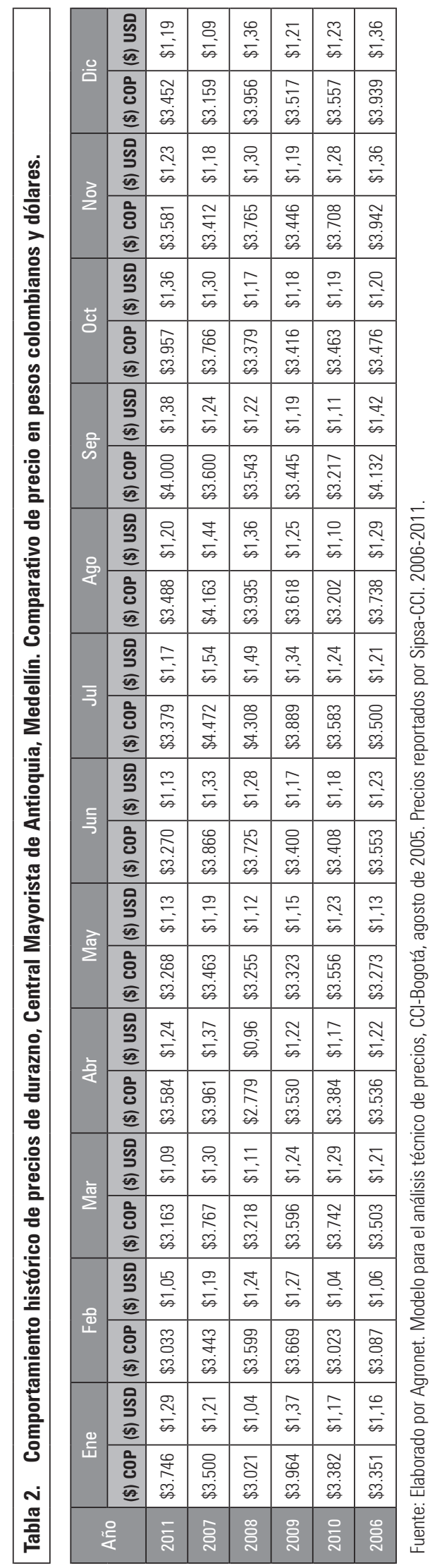




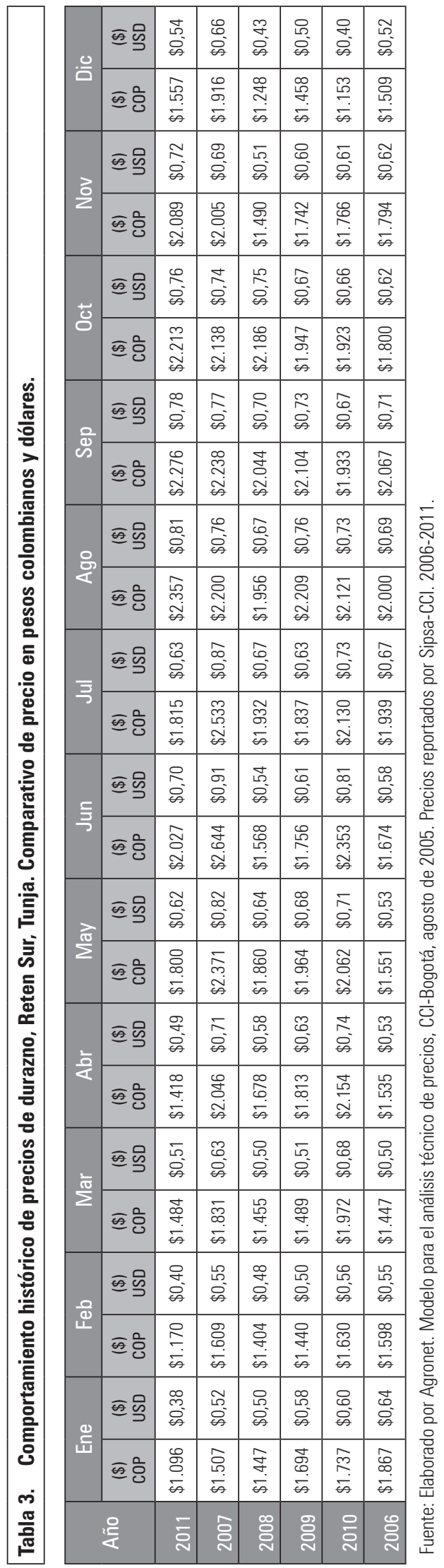

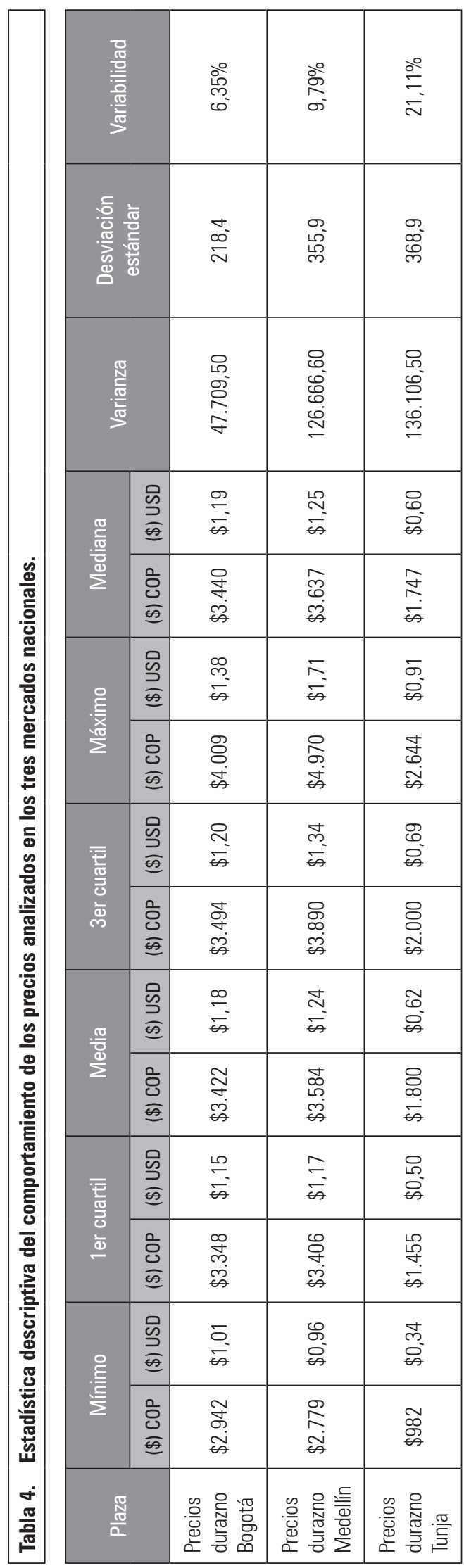


más bajo, en relación a los mercados más distantes, en los cuales el precio es más elevado.

Relacionado con la tendencia y estacionalidad de precios de durazno, en una primera instancia la labor del análisis estuvo centrada en graficar la serie de tiempo de los precios del fruto y explorarla mediante AES y Método de Loess, identificando que el comportamiento estacional y de tendencia no están presentes en los mercados de Corabastos, Bogotá y Central Mayoristas de Antioquia en Medellín, comportándose de manera diferenciada el mercado de Reten Sur de Tunja donde hay presencia de estacionalidad no muy definida y no hay tendencia clara.

Tanto por AES como por el método de Loess, ratifican el comportamiento identificado en la modelación de la serie de tiempo para los tres mercados seleccionados, en la ilustración. En el análisis serie precio durazno por medio del método de Loess, se constata la presencia de ruido en las gráficas de estacionalidad, especialmente en los mercados de Bogotá y Medellín, siendo menor en el mercado de Tunja, tal como se aprecia en la figura 2. Para el caso del precio del durazno, el método de Loess identifica una tendencia definida en los tres mercados analizados, hallando estacionalidad únicamente en el mercado de Reten Sur de Tunja, a diferencia de los mercados de Medellín y Bogotá los cuales poseen un comportamiento no estacional y no se observan comportamientos cíclicos.

A continuación, se muestran los modelos ajustados propuestos para explicar el comportamiento de los precios del durazno en cada una de las centrales.

Los modelos propuestos se ajustaron mediante la función $<<$ auto.arima $>>$ que busca entre los diferentes valores de los parámetros $p, q, \mathrm{y} d$, tanto para la parte regular como para la parte estacional, el modelo más adecuado. Esta función emplea el algoritmo de (Hyndman y Khandakar, 2008), que elige los parámetros minimizando el $A I C$, y los valores de $d$ y $D$ mediante test de raíz unitaria (García et al., 2013).

En cada uno de los modelos se evaluó el supuesto de ruido blanco mediante la prueba de Ljung-Box. Para los precios del durazno en Bogotá los resultados fueron: $0,5079, \mathrm{df}=1$, valor $\mathrm{P}=0,4761$. En el caso de Medellín: 0,1707, df $=1$, valor $\mathrm{P}=0,6795$. Finalmente, para el caso de Tunja: $14,0107, \mathrm{df}=12$, valor $\mathrm{P}=0,3$. En cada modelo, se valida el supuesto de ruido blanco y se comprueba que los residuos no conservan una estructura que deba ser explica por un modelo más complejo.

En general, se puede concluir que en Bogotá y Medellín, las series no evidente la tendencia y no se aprecia estacionalidad. Se concluye que los precios de Tunja no tienen tendencia, pero tienen estacionalidad anual.

\section{Volatilidad en precios de durazno}

En el análisis posterior se concentró en la identificación de la presencia o ausencia de volatilidad en las series de tiempo para los mercados seleccionados, para esto se analizaron los correlogramas de los residuales al cuadrado de cada modelo propuesto, comprobándose que no existe una clara evidencia de la volatilidad, ya que los residuos al cuadrado no se correlacionan en la mayoría de los retrasos en ninguno de las series de tiempo de los dos productos analizados.

No se observa presencia recurrente de shocks de mercados para ninguno de los mercados analizados. Al explorar las posibles causas del por qué no predomina la estacionalidad y volatilidad dentro de las series de tiempo de los precios de durazno, se hace relevante enunciar los siguientes hechos. Para Fischer et al. (2012) la implementación de paquetes tecnológicos en la producción de durazno con la técnica de cosecha forzada ha permitido la recolección de fruta durante todo el año, de igual manera, se han incrementado las áreas de producción en el país, garantizando una provisión constante en los mercados más grandes, evitando que se presenten periodos de escases.

Este grupo de frutas poseen elasticidades altas lo cual indica que a mayores cambios en el precio varía el volumen demandado y ofertado, ya que los consumidores suplen sus necesidades con productos sustitutos (Andreyeva et al., 2010), este es el caso del durazno

Tabla 5. Modelos ajustados propuestos para explicar el comportamiento de los precios del durazno en cada una de las centrales.

\begin{tabular}{|c|c|c|}
\hline Tunja & Medellín & Bogotá \\
\hline ARIMA $(1,0,0) \times(0,1,1)_{[12]}$ & AR(1) & ARIMA $(1,0,1)$ \\
AIC: 1820.37 & AIC: 1555.04 & AIC: 781.12 \\
\hline
\end{tabular}


donde los precios no presentan shocks, ya que las fuerzas de mercado regulan el precio.

\section{CONCLUSIONES}

El principal aporte del presente estudio es la aplicación de modelos econométricos, que analizan el comportamiento histórico de las series de tiempo de precios de mercados mayoristas en durazno, los cuales se pueden emplear para la elaboración de modelos predictivos de ingresos en otros cultivos, dotando de una herramienta necesaria en la evaluación financiera de proyectos en cultivos de tardío rendimiento.

Otra contribución importante del estudio es el uso de los tres modelos desarrollados, dado que se pueden construir modelaciones predictivas del comportamiento a futuro de los precios de durazno en los mercados evaluados, con la seguridad de que si un productor indaga sobre cuál será el precio del kg de durazno para un proyecto de inversión, se le puede responder cual será el rango de precios para los próximos años con un alto grado de confiabilidad utilizando esta metodología.

La modelación de comportamientos de los mercados mayoristas aplicada a la gestión de las agroempresas, facilita el desarrollo de estrategias organizacionales adecuadas a los mercados locales, permitiendo realizar evaluaciones financieras más ajustadas haciendo más competitiva la agricultura colombiana.

Al conocer que el precio del durazno no presenta estacionalidad, ni volatilidad en los mercados mayoristas estudiados (Fig. 3), se genera un elemento a favor para que los pequeños productores, consideren estos destinos para el producto, con la implementación de tecnologías poscosecha que prolonguen la vida útil del mismo, así como la elaboración de trabajos en diseños de nuevos empaques y desarrollo de la cadena de frio, de la misma manera, se sugiere adelantar proyectos que midan el riesgo climático, mercados, sociales en inversiones de cultivos de caducifolios.

Entendiendo que la volatilidad de los precios mide la variación o fluctuación de los precios en un periodo

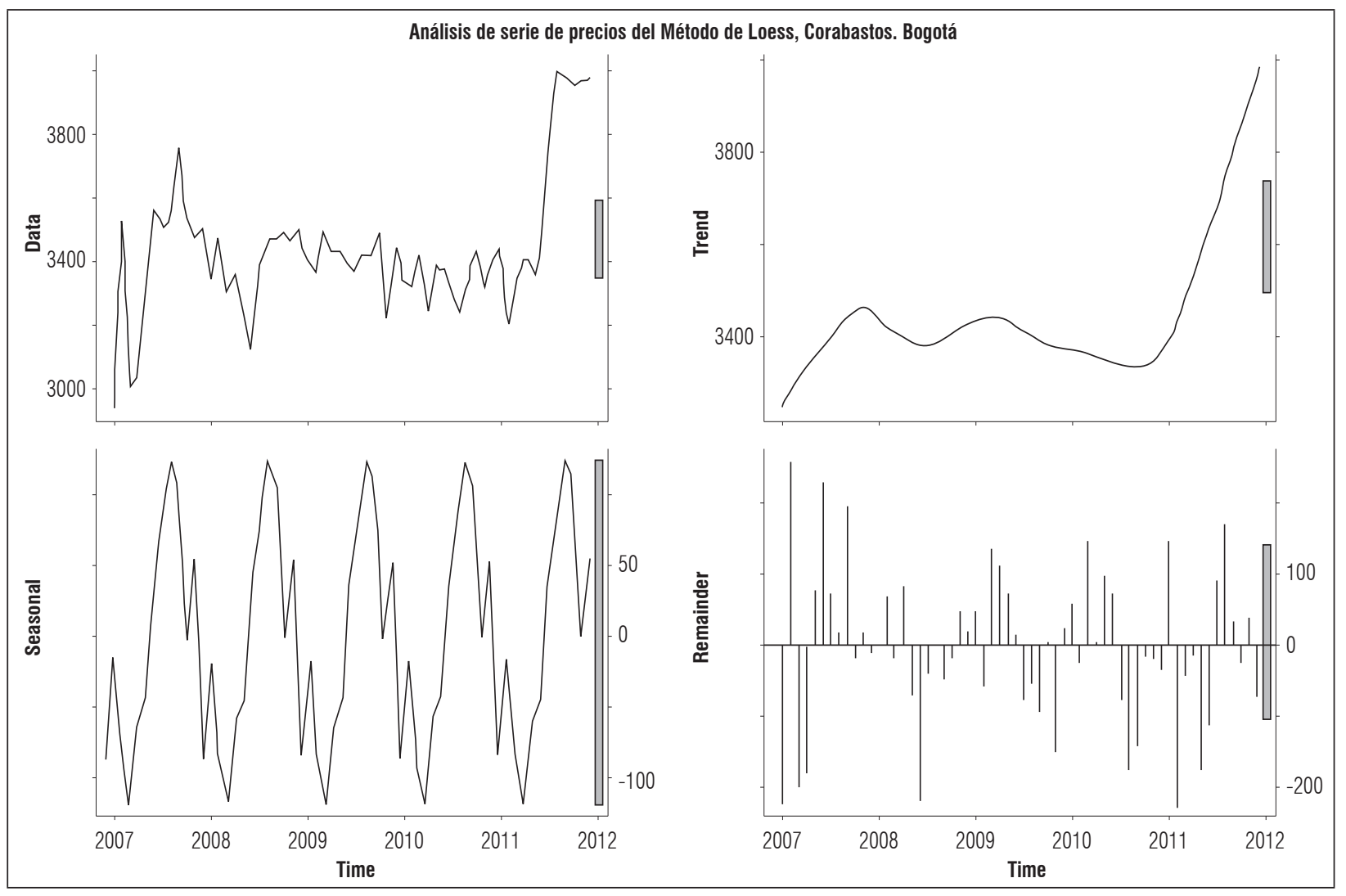

Figura 3. Análisis de serie de precio durazno por medio del Método de Loess en los tres mercados analizados en el periodo 2006 a 2011. 


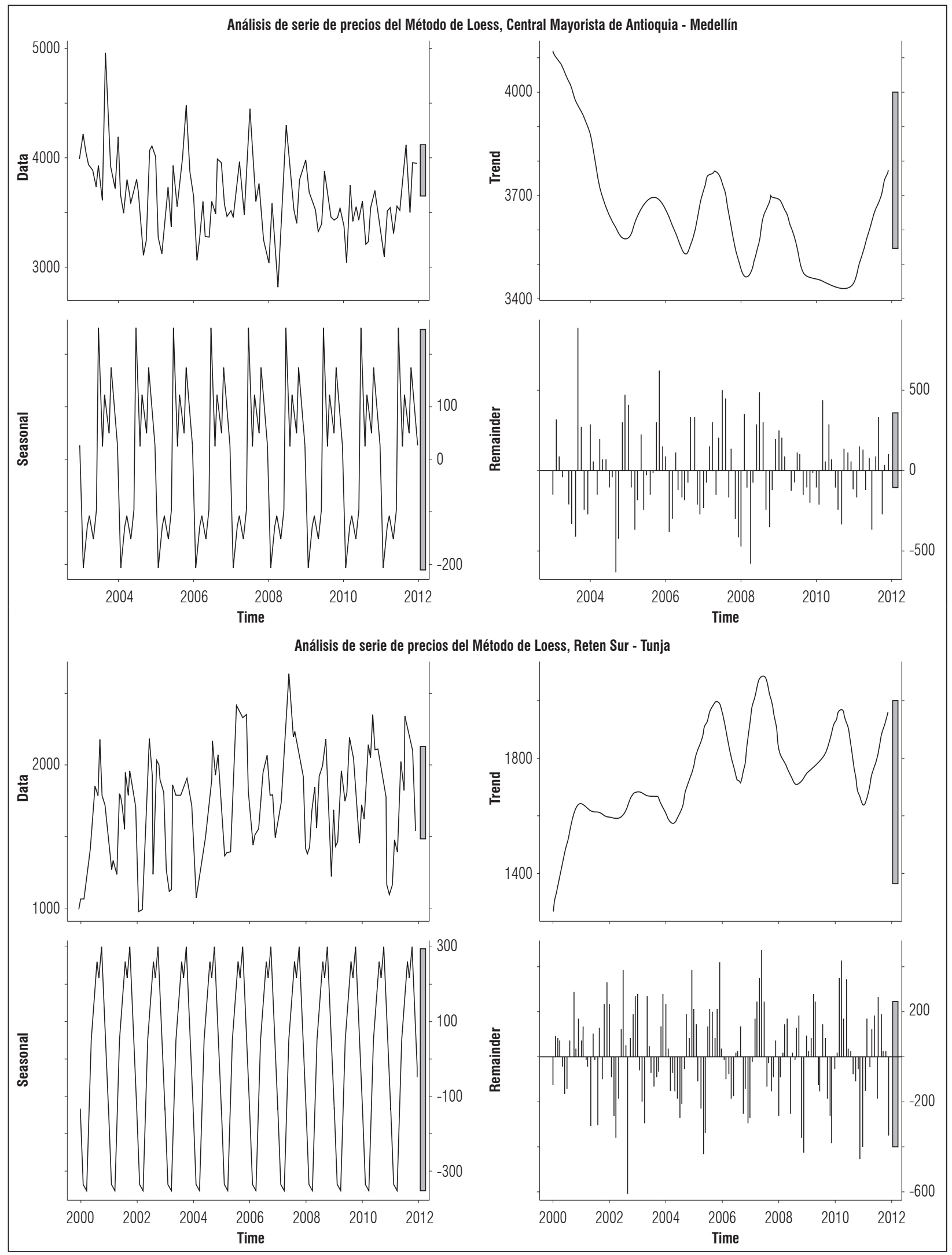

Figura 3. Análisis de serie de precio de durazno por medio del Método de Loess en los tres mercados analizados en el periodo 2006 a 2011. 


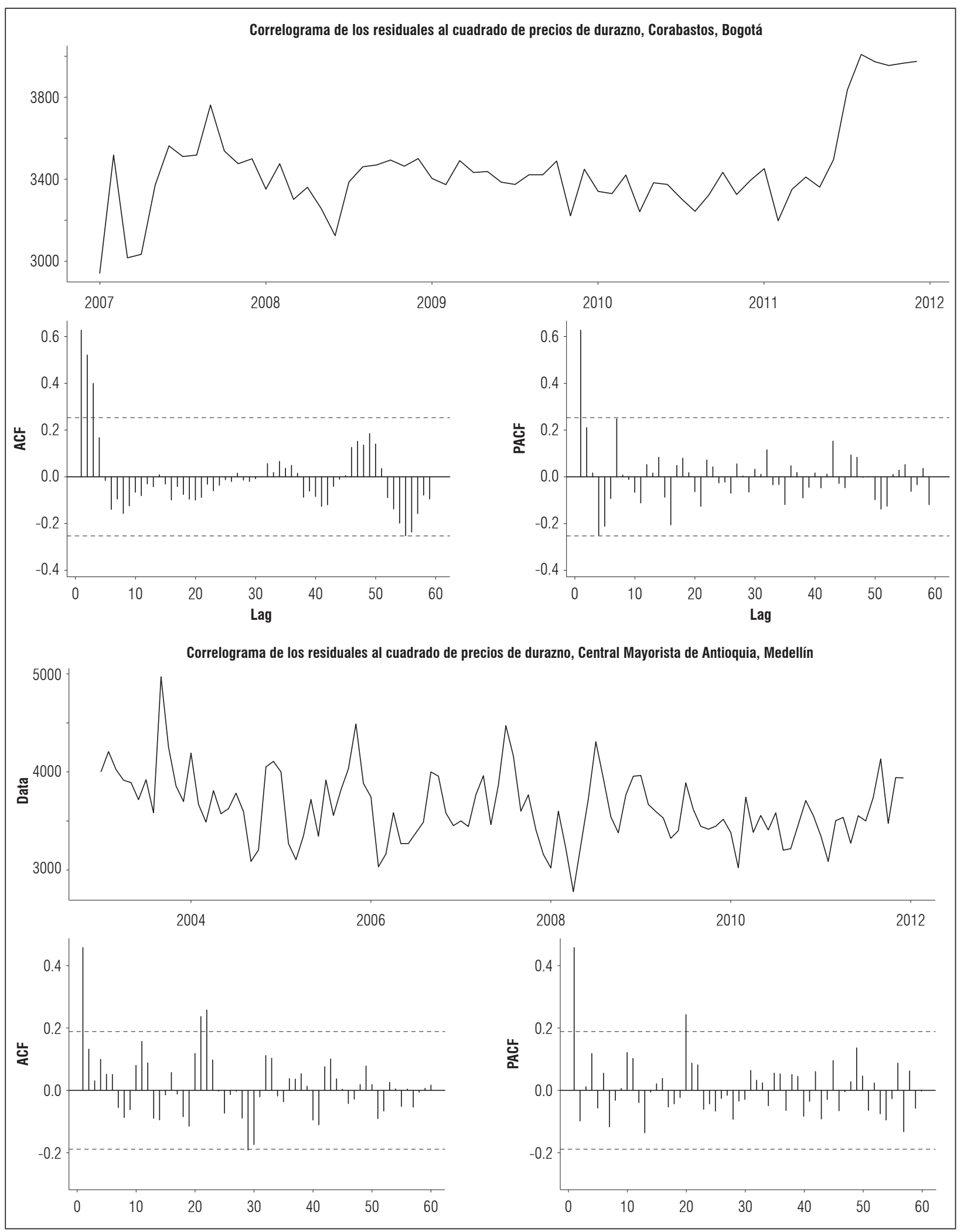

Figura 4. Analisis comparativo de correlograma de residuales al auadrado de precios de durazno, donde no se evidencia la asusencia de volatilidad en los mercados analizados. 


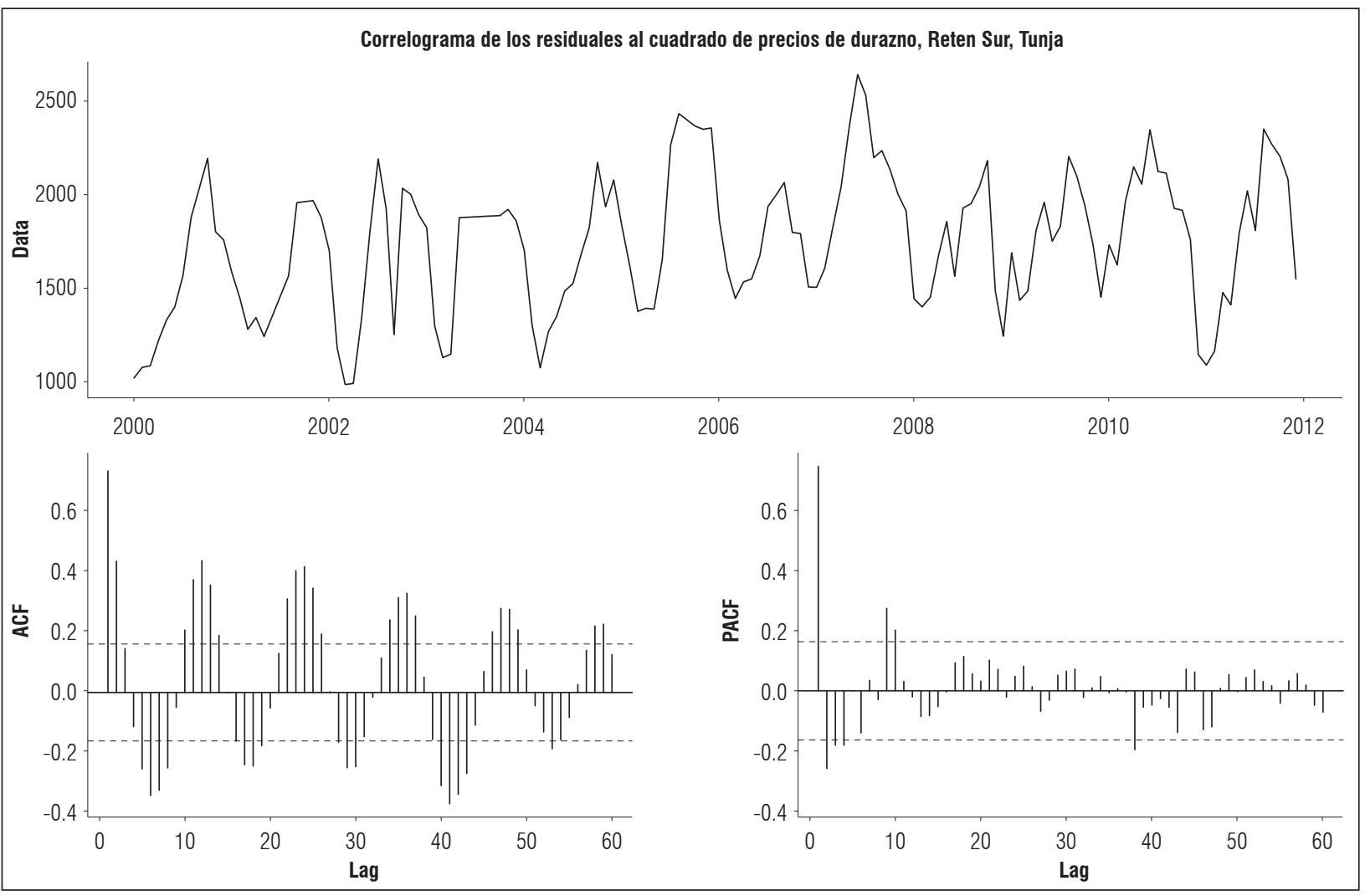

Figura 4. Analisis comparativo de correlograma de residuales al auadrado de precios de durazno, donde no se evidencia la asusencia de volatilidad en los mercados analizados.

de tiempo (FAO, 2012), se hace una reflexión de cómo estas frutas en seis años no han tenido verdaderos incrementos en los precios de mercado, como se presenta en la (Fig. 4). Correlograma de Residuales al Cuadrado de Precios de Mercado. Aspecto que contrasta con los altos costos de producción, llevando a la reducción de los ingresos y por ende a disminuir las utilidades de los empresarios.

\section{REFERENCIAS BIBLIOGRÁFICAS}

Agronet y SIPSA. 2015. Modelo para el análisis técnico de precios - CCI, Bogotá, agosto de 2005. Precios reportados por Sipsa-CCI 2006-2011. Bogotá, Colombia.

Aimin, H. 2010. Uncertainty, risk aversion and risk management in agriculture. Agr. Agric. Sci. Procedia 1, 152156. Doi: 10.1016/j.aaspro.2010.09.018

Andreyeva, T., M.W Long y K.D.Brownell. 2010. The impact of food prices on consumption: a systematic review of research on the price elasticity of demand for food. Amer. J. Pub. Health, 100(2), 216-222. Doi: 10.2105/AJPH.2008.151415

Balcombe, K. 2009. The nature and determinants of volatility in agricultural prices: An empirical study from 1962-2008. University of Reading, Reading, UK.

Baugher, A. 2003. Anatomy and taxonomy. pp. 3-8. En: Baugher, A. y S. Singha (eds.). Concise encyclopedia of temperate tree fruit. Food Products Press, New York, NY.

Bellemare, M.F. 2015. Rising food prices, food price volatility, and social unrest. Amer. J. Agric. Econ. 97(1), 1-21. Doi: 10.1093/ajae/aau038

Bollerslev, T. 1986. Generalized autoregressive conditional heteroskedasticity. J. Econometr. 31(3), 307-327. Doi: 10.1016/0304-4076(86)90063-1

Cárdenas, J. y G. Fischer. 2013. Clasificación botánica y morfología. pp. 21-29. En: Miranda, D., G. Fischer y C. Carranza (eds.). Los frutales caducifolios en Colombia. Situación actual, sistemas de cultivo y plan de 
desarrollo. Sociedad Colombiana de Ciencias Hortícolas, Bogotá, Colombia

Castro, A. y G.A. Puentes. 2012. Ciruelo (Prunus salicina Lindl.) y duraznero (Prunus persica (L.) Batsch.). pp. 370-392. En: Fischer, G. (ed.). Manual para el cultivo de frutales en el trópico. Produmedios, Bogotá, Colombia.

CEPAL, FAO e IICA. 2011. Volatilidad de precios en los mercados agrícolas (2000-2010): Implicaciones para América Latina y opciones de políticas. Boletín CEPAL/ FAO/IICA Perspectivas de la agricultura y del desarrollo rural en las Américas: una mirada hacia América Latina y el Caribe 3, 1-36.

CFS, C.d.S.A.M. 2011. Volatilidad de los precios y seguridad alimentaria. Un informe del grupo de expertos de alto nivel sobre seguridad alimentaria y nutrición. Capítulo 1: Comportamiento reciente de los precios internacionales de alimentos: Tres interpretaciones. Vol. 1. Editorial HLPE, Roma-Italia. pp. 19-37.

Cooper, R., R, Lawrence, B. Bosworth y H. Houthakker. 1975. The 1972-75 commodity boom. Brook. Papers Econ. Act. 3, 671-723. Doi: 10.2307/2534151

Doporto M.I. y G. Michelena. 2011. La volatilidad de los precios de los commodities: el caso de los productos agrícolas. Rev. Centro Econ. Int. (CEI) 1, 7-27.

Engle, R. 1982. Autoregressive conditional heteroscedasticity with estimates of the variance of United Kingdom inflation. Econometrica: J. Econ. Soc. 50(4), 987-1007. Doi: $10.2307 / 1912773$

Fachinello, J.M., J. Pasa, J. Schmtiz y D. Betemps. 2011. Situação e perspectivas da fruticultura de clima temperado no Brasil. Rev. Bras. Frutic. 33(1), 109-120. Doi: 10.1590/S0100-29452011000500014

FAO. 2006. Seguridad alimentaria. Informes de Política 2. Roma, Italia.

FAO. 2010. La volatilidad de precios en los mercados agrícolas. Perspectivas económicas y sociales. Informes de Política 12. Roma, Italia.

FAO. 2012. Volatilidad de los precios desde una perspectiva mundial. Documento técnico de referencia para la reunión de alto nivel sobre: La volatilidad de los precios de los alimentos y el papel de la especulación. Roma, Italia.

FAOSTAT. 2012. Statistical databases. Food and Agriculture Organization of the United Nations, Roma, Italia.

Fischer, G., F. Casierra-Posada y C. Villamizar. 2010. Producción forzada de duraznero (Prunus persica (L.) Batsch) en el altiplano tropical de Boyacá (Colombia). Rev. Colomb. Cienc. Hortíc. 4(1), 19-32. Doi: 10.17584/ rcch.2010v4i1.1223

Gilbert, C. y C. Morgan. 2010. Food price volatility. Phil. Trans. Royal Soc.London, B. Biol. Sci. 365(1554), 30233034. Doi: 10.1098/rstb.2010.0139

Giraldo, N. 2006. Series de tiempo con R. 1-157. Universidad Nacional de Colombia, Medellín, Colombia.

García R.E., C. Guisande, D.J. González, J. Heine, P. Pelayo, A. Manjarrás y C. Granado. 2013. ModestR: a software tool for managing and analyzing species distribution map databases. Ecography 36(11), 1202-1207. Doi: 10.1111/j.1600-0587.2013.00374.x

Hyndman, R. y Y. Khandakar. 2008. Automatic time series forecasting: the forecast package for R. J. Stat. Software 27(3), 1-22. Doi: 10.18637/jss.v027.i03

Minagricultura. 2012. Frutales por departamentos. pp. 57-58. Departamento y sus frutas. Pp. 156. Anuario estadístico de frutas y hortalizas 2007-2011 y sus calendarios de siembras y cosechas. Ministeria de Agricultura y Desarrollo Rural, Bogotá. Editorial JL Impresores Ltda., Bogotá, Colombia.

Miranda, D., G. Fischer y C. Carranza. 2013. Los frutales caducifolios en Colombia. Situación actual, sistemas de cultivo y plan de desarrollo. Sociedad Colombiana de Ciencias Hortícolas, Bogotá, Colombia

Pérez, C. 2006. Econometría de las series temporales. Pearson Prentice Hall, Madrid, España.

Puentes, G. 2006. Sistema de producción de frutales caducifolios en el departamento de Boyacá. Equidad Desarro. 5, 39-46.

Puentes, G., L. Rodríguez y L. Bermúdez. 2008. Análisis de grupo de las empresas productoras de frutales caducifolios del departamento de Boyacá. Agron. Colomb. 26(1), 146-154.

Ramírez, A., S.F. Juárez y F.J. Ariza. 2012. Análisis espectral singular de series de tiempo - aplicaciones del análisis espectral singular. Editorial Académica Española, Madrid, España.

Taušer, J. y R. Čajka. 2014. Hedging techniques in commodity risk management. Agric. Econ. 60, 174-182.

Taylor, S.J. 2008. Modelling financial time series. $2^{\text {nd }}$ ed. World Scientific Publishing, Singapore.

Westwood, M.N. 1993. Temperate-zone pomology. 3a ed. Timper Press, Oregon, OR. 